\title{
Findings Annihilator(s) via Fault Injection Attack (FIA) on Boolean Function of Grain v0
}

\author{
Muhammad Rezal Kamel Ariffin1, 2,Wan Zariman Omar@Othman1,3,*, Solahuddin Shamsuddin3, Zahari \\ Mahad1, and Suhairi Mohd Jawi3 \\ 1 Laboratory of Cryptography, Analysis and Structure, Institute for Mathematical Research, Universiti Putra Malaysia \\ 2 Department of Mathematics, Faculty of Science, Universiti Putra Malaysia (UPM), Selangor, Malaysia \\ ${ }^{3}$ CyberSecurity Malaysia
}

\begin{abstract}
In developing stream cipher algorithms, Boolean function is one of vital elements. Attacks on LFSR-based stream cipher is the challenge for the cryptanalyst to get low-degree annihilator(s). In this paper, we proposed Fault Injection Attack (FIA) on Boolean function of Grain v0, which is the original variant of Grain family algorithm. Fault injection attack (FIA) is used on Boolean function of Grain v0 by replacing certain coefficient with value of one (1) which results in the generation of several injected Boolean functions. With these injected Boolean function, we proceed using HAO's algorithm to find annihilator(s). As a result, we obtained several new annihilator(s) of Grain v0's Boolean function. This new annihilator(s) will be utilized to launch algebraic attacks upon Grain v0.
\end{abstract}

\section{Introduction}

Cryptology is science that incorporates both cryptography and cryptanalysis. Cryptography is divided into two types, which are symmetric cryptography and asymmetric cryptography. In symmetric cryptography, only one key namely secret key will be used to encrypting and decrypting the data. Meanwhile for the asymmetric cryptography, a key pair will be used to encrypting and decrypting the data which namely public key and private key. The symmetric cryptography can be divided into two types symmetric cipher, which are block cipher and stream cipher. In stream cipher, we have three (3) class [1];

I. The one-time pad

1. Definition Let Vernam cipher over the binary alphabet is defined by $\mathrm{c}_{\mathrm{i}}=\mathrm{m}_{\mathrm{i}} \oplus \mathrm{k}_{\mathrm{i}}$ for $i=1,2,3, \ldots$, where $m_{1}$

II. Synchronous Stream Ciphers

2. Definition A synchronous stream cipher is one in which the keystream is generated independently of the plaintext message and of the ciphertext.

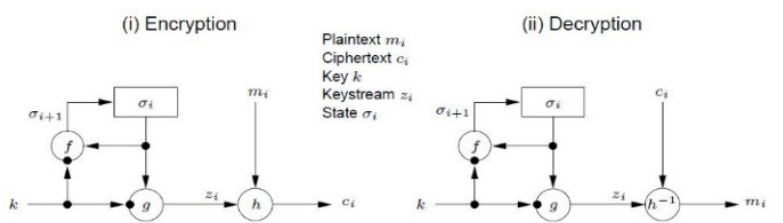

Figure 1: General model of a synchronous stream cipher

In a synchronous stream cipher a stream of pseudo-random digits is generated independently of the plaintext and ciphertext messages, and then combined with the plaintext (to encrypt) or the ciphertext (to decrypt) (refer to Figure 1). In the most common form, binary digits are used (bits), and the keystream is combined with the plaintext using the exclusive or operation (XoR). This is termed a binary additive stream cipher (refer to Figure 2).

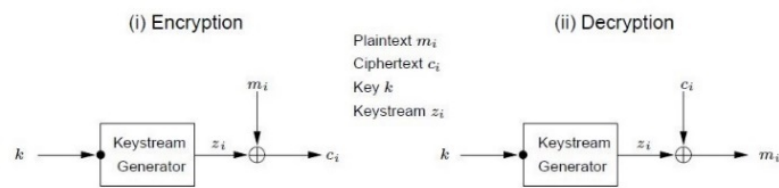

Figure 2: General model of a binary additive stream cipher (above)

In a synchronous stream cipher, the sender and receiver must be exactly in step for decryption to be successful. If digits are added or removed from the message during transmission, synchronisation is lost. To restore synchronisation, various offsets can be tried systematically to obtain the correct decryption. Another approach is to tag the ciphertext with markers at regular points in the output.

\section{Self-synchronous stream ciphers}

3. Definition A self-synchronous or asynchronous stream cipher is one in which the keystream is generated as function of the key and a fixed number of previous ciphertext digits.

The encryption function of self-synchronous stream cipher can be described by the equations:

$$
\begin{aligned}
\sigma_{i} & =\left(c_{i-t}, c_{i-t+1}, \ldots, c_{i-1}\right) \\
z_{i} & =g\left(\sigma_{i}, k\right) \\
c_{i} & =h\left(z_{i}, m_{i}\right)
\end{aligned}
$$

where $\sigma_{i}=\left(c_{i-t}, c_{i-t+1}, \ldots, c_{i-1}\right)$ is the initial state, $k$ (non-secret) $\mathrm{g}$ is the function which produces the key stream, $z_{i}$ and $h$ is the output function which combines the keystream and plaintext $m_{i}$ to produces ciphertext $c_{i}$. Please refer to Figure 3 . 


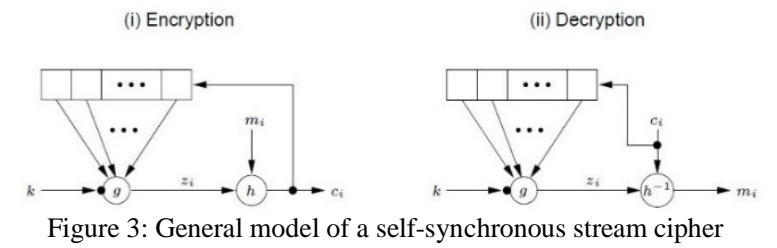

For each type of ciphers, it has their own types of attacks. For example of the symmetric stream cipher, there are many types of attacks such as:

1. Algebraic Attack

2. Exhaustive Search Attack

3. Correlation Attack

4. Fault Attack (including Fault Injection)

5. Distinguishing Attack

6. Chosen-IV attack

7. Slide Attack

8. Cube Attack

9. Guess and Determine Attack

In this paper, we will focus more on symmetric stream cipher namely Fault Injection Attack (FIA), which is specifically for Boolean function of stream cipher algorithm.

\section{Description of Grain v0}

Stream cipher can be classified into two types, which are synchronous stream ciphers, and asynchronous stream ciphers also known as self-synchronous stream ciphers.

Grain is a stream cipher primitive that was designed by Hell et al in 2007 to be very easy and small to implement in hardware. Grain v0 is a bit oriented synchronous stream cipher and the key stream is generated independently from plain text and was designed based on two shift registers, one (1) with linear feedback shift register (LFSR) and one with nonlinear feedback shift register (NFSR). Both shift registers are 80-bits, the key size is 80-bits and the IV size is 64-bits. Grain v0 consists of LFSR denoted by

$$
S_{i}, S_{i+1}, \ldots, S_{i+79}
$$

and NFSR is denoted by

$$
b_{i}, b_{i_{+1}}, \ldots, b_{i+79} \text {. }
$$

Meanwhile LFSR function denoted as:

$$
f(x)=1+x^{18}+x^{29}+x^{42}+x^{57}+x^{67}+x^{80}
$$

and to remove any possible ambiguity, update function of the LFSR is defined as:

$$
s_{i+80}=s_{i+62}+s_{i+51}+s_{i+38}+s_{i+23}+s_{i+13}+s_{i}
$$

NFSR of Grain v0, $g(x)$ is defined as:

$$
\begin{gathered}
g(x)=1+x^{17}+x^{20}+x^{28}+x^{35}+x^{43}+x^{47}+x^{52}+x^{59}+x^{65}+x^{71}+ \\
x^{80}+x^{17} x^{20}+x^{43} x^{47}+x^{65} x^{71}+x^{20} x^{28} x^{35}+x^{47} x^{52} x^{59}+ \\
x^{17} x^{35} x^{52} x^{71}+x^{20} x^{28} x^{43} x^{47}+x^{17} x^{20} x^{59} x^{65}+x^{17} x^{20} x^{28} x^{35} x^{43}+ \\
x^{47} x^{52} x^{59} x^{65} x^{71} \\
+
\end{gathered}
$$

and to remove any possible ambiguity, update function of the NFSR is defined as:

$$
\begin{aligned}
b_{i+80}= & s_{i}+b_{i+63}+b_{i+60}+b_{i+52}+b_{i+45}+b_{i+37}+b_{i+33}+b_{i+28}+b_{i+21}+ \\
& b_{i+15}+b_{i+9}+b_{i}+b_{i+63} b_{i+60}+b_{i+37} b_{i+33}+b_{i+15} b_{i+9}+ \\
& b_{i+60} b_{i+52} b_{i+45}+b_{i+33} b_{i+28} b_{i+21}+b_{i+63} b_{i+45} b_{i+28} b_{i+9}+
\end{aligned}
$$

$$
\begin{aligned}
& b_{i+60} b_{i+52} b_{i+37} b_{i+33}+b_{i+63} b_{i+60} b_{i+21} b_{i+15} \\
& b_{i+63} b_{i+60} b_{i+52} b_{i+45} b_{i+37}+b_{i+33} b_{i+28} b_{i+21} b_{i+15} b_{i+9} \\
& b_{i+52} b_{i+45} b_{i+37} b_{i+33} b_{i+28} b_{i+21}
\end{aligned}+
$$

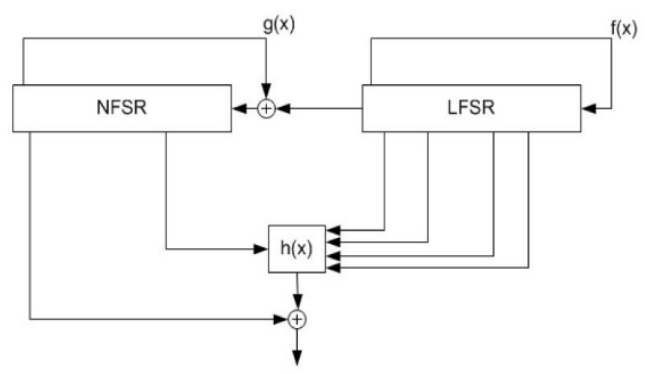

Figure 4: Grain v0 diagram.

Figure 4 show diagram of both two shift registers represent state of $h$ : algorithm. Four (4) variables taken from LFSR and one (1) variable en from NFSR as input to a Boolean function, $h(x)$. This function has algebraic degree 3 . This Boolean function is defined as:

$$
\begin{aligned}
& h(x)=x_{1}+x_{4}+x_{0} x_{3}+x_{2} x_{3}+x_{3} x_{4}+x_{0} x_{1} x_{2}+x_{0} x_{2} x_{3}+x_{0} x_{2} x_{4}+x_{1} x_{2} x_{4}+ \\
& x_{2} x_{3} x_{4}
\end{aligned}
$$

where the variables $\mathrm{x}_{0}, \mathrm{x}_{1}, \mathrm{x}_{2}, \mathrm{x}_{3}$ and $\mathrm{x}_{4}$ corresponds to the tap positions of $s_{i+3}, s_{i+25}, s_{i+46}, s_{i+64}$ and $b_{i+63}$ respectively. Output of this filter function is masked with the bit, $b_{i}$ from the NFSR to produce keystream.

\section{Basic study on Boolean function}

4 Definition (Boolean function) A Boolean function $\mathrm{f}$ on $\mathrm{n}$ variables is a mapping from $\{0,1\} \mathrm{n}$ to $\{0,1\}$

5 Definition (Algebraic normal form (ANF) of Boolean function) Every Boolean function $f$ can be expressed as a multivariate polynomial over $F_{2}$. This polynomial is known as algebraic normal form of the Boolean function $h$. The general form of algebraic normal form of $h$ is given by:

$$
f\left(x_{1}, \ldots, x_{n}\right)=a_{0} \oplus \bigoplus_{1 \leq i \leq n} a_{i} x_{i} \oplus \bigoplus_{1 \leq i<j \leq n} a_{i j} x_{i} x_{j} \oplus \cdots \oplus a_{12} \ldots{ }_{n} x_{1} x_{2} \cdots x_{n} .
$$

6 Definition (Degree of Boolean function) Degree of a Boolean function $h$ is defined as $\operatorname{deg}(h)=$ number of variables in the highest order product term in the algebraic normal form of $h$. Functions of degree at most one are called affine function. An affine function with constant term equal to zero is called linear function. For example, Grain v0's Boolean function has $\operatorname{deg}(h)=3$.

7 Definition (Annihilator of a Boolean function) A non-zero Boolean function $g$ of $n$-variables is said to be a annihilator of a Boolean function $f$ iff $g(X) \cdot f(X)=0, \forall X \in\{0,1\}^{n}$.

\section{Definition of our Fault Injection Attack (FIA) on Grain v0's} Boolean Function

The attacker is assumed able to inject exactly one bit ( 1 or 0 ) in any one of active coefficients in the Boolean function [3][4]. In Grain v0, Boolean function is defined as:

$$
h(x)=x_{1}+x_{4}+x_{0} x_{3}+x_{2} x_{3}+x_{3} x_{4}+x_{0} x_{1} x_{2}+x_{0} x_{2} x_{3}+x_{0} x_{2} x_{4}+x_{1} x_{2} x_{4}+
$$

This paper will inject value of one (1) in each active coefficients of Grain v0’s Boolean function. For example, in Grain v0’s Boolean function, we get nineteen (19) active coefficients such as below:

$\begin{array}{ll}\text { 1. } & x_{0} \\ \text { 2. } & x_{1} \\ \text { 3. } & x_{2} \\ \text { 4. } & x_{3}\end{array}$ 

5. $x_{4}$
6. $x_{0} x_{1}$
7. $x_{0} x_{2}$
8. $x_{0} x_{3}$
9. $x_{0} x_{4}$
10. $x_{1} x_{2}$
11. $x_{1} x_{4}$
12. $x_{2} x_{3}$
13. $x_{2} x_{4}$
14. $x_{3} x_{4}$
15. $x_{0} x_{1} x_{2}$
16. $x_{0} x_{2} x_{3}$
17. $x_{0} x_{2} X_{4}$
18. $x_{1} X_{2} x_{4}$
19. $x_{2} X_{3} x_{4}$

With these nineteen $(i=19)$ active coefficients so we have nineteen (19) new injected Boolean function. So let new injected Boolean function defined as $h_{i}(x)$. From all new derived Boolean function, we will use $H A O$ 's algorithm to find annihilator(s) of the current/injected Boolean function. For example, we inject with bit-1 into active coefficients $x_{0}$ so we will get from;

$h(x)=x_{1}+x_{4}+x_{0} x_{3}+x_{2} x_{3}+x_{3} x_{4}+x_{0} x_{1} x_{2}+x_{0} x_{2} x_{3}+x_{0} x_{2} x_{4}+$ $x_{1} x_{2} x_{4}+x_{2} x_{3} x_{4}$

$\Rightarrow x_{1}+x_{4}+x_{3}+x_{1} x_{2}+x_{2} x_{4}+x_{3} x_{4}+x_{1} x_{2} x_{4}+x_{2} x_{3} x_{4}$

\section{HAO's Method}

From Courtois and Meier, the key step of algebraic attack (stream cipher) is to build low-degree over-defined equations, and this method can be enhanced to find low-degree annihilator(s) of combination of the keystream generator[5]. In HAO's paper, there is three (3) algorithms/methods to find annihilator(s) of the given Boolean function [6].

In this paper, we will use algorithm 2 in HAO's algorithm to find annihilator of the injected Grain v0's Boolean function. We will consider all the n-variable non-zero monomials of degree $\leq \mathrm{d}$, denoted by $A_{d}=\{1$, $x 1, x 2, \ldots, x n, x 1 x 2, x 1 x 3, x 2 \times 3, x 1 x 4, x 2 x 4, x 3 x 4, \ldots, x n-1 x n, \ldots, x n-d+1$

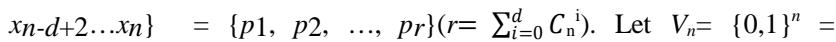
$\left\{v_{1}, v_{2}, \ldots, v_{r}\right\}$, where $r=2^{n}$ and the ordering among the vectors is considered in lexicographic ordering.

Then, we denote $\mathrm{C}=\left\{p \bullet h \mid p \in A_{d}\right\}=\left\{h p_{1}, h p_{2}, h p_{3}, \ldots, h p_{r}\right\}$. For any $h \in B_{n}$, we denote it by a $2^{\mathrm{n}}-$ tuple vector $\left\{h_{1}, h_{2}, h_{3}, \ldots, h_{r} n\right\}$.

For example, Boolean function for Grain v0 is defined as:

$h(x)=x_{1}+x_{4}+x_{0} x_{3}+x_{2} x_{3}+x_{3} x_{4}+x_{0} x_{1} x_{2}+x_{0} x_{2} x_{3}+x_{0} x_{2} x_{4}+x_{1} x_{2} x_{4}$ $+x_{2} x_{3} x_{4}$

can be represented by $(0, \mathbf{1}, \mathbf{0}, \mathbf{0}, \mathbf{1}, \mathbf{0}, \mathbf{0}, \mathbf{1}, \mathbf{0}, \mathbf{0}, \mathbf{0}, \mathbf{0}, \mathbf{1}, \mathbf{0}, \mathbf{1}, \mathbf{1}, \mathbf{0}$, $0,1,1,0,0,1,0,1,0,0,0,0,0,0)$.

Algorithm 2 (as in [6]) is defined as below:

Algorithm Given a n-variable Boolean function $h$, find all annihilator(s) of $h$ with degree $\leq \mathrm{d}$.

Step 1: Construct matrix $M_{d}(h)$.

Step 2: Convert $M_{d}(h)$ into row ladder matrix $M_{d}(h) *$ using Gaussian elimination.

Step 3: If there exits zero-rows in $M_{d}(h)^{*}$, it certainly exists a annihilator $g$ of $h$, and obtain $g$ by using the inverse procession of Step 2, or else, there is no annihilator of $h$ with degree $\leq d$.

Theorem $1|C|<\left|A_{d}\right| \Rightarrow$ There exists at least one (1) annihilator of $h$ with degree $\leq d$.
Proof. $|C|<\left|A_{d}\right| \Leftrightarrow \exists p_{1}, p_{2} \in \mid A_{d}$ with $p_{1} \neq p_{2}$ such that $p_{1} \bullet h=p_{2} \bullet h \Leftrightarrow$ $h \bullet\left(p_{1}+p_{2}\right)=0 \Rightarrow p_{1}+p_{2}$ is an annihilator of $h$ with degree $\leq d$.

Theorem 2 There exists annihilator of $h$ with degree $\leq d \Leftrightarrow$ rank $\left(M_{d}(h)\right)<\left|A_{d}\right|$

Proof. There exists annihilator $g$ of $h$ with degree $\leq d \Leftrightarrow h(x) \bullet g$ $(x)=0$ for any $x \in\{0,1\} n \Leftrightarrow$ The sum of rows in $M_{d}(h)$ corresponding to the terms of $g$ is zero $\Leftrightarrow$ rank $\left(M_{d}(h)\right)<$ The number of rows in $M_{d}(h) \Leftrightarrow$ rank $\left(M_{d}(h)\right)<\left|A_{d}\right|$.

\section{Analysis}

The adversary is assumed able to inject exactly one bit (bit 1) in any one of active coefficients in the Boolean function. In this paper, we will inject value of one (bit-1) into Grain v0's Boolean function. All new injected Boolean function is defined as Table 1:

\begin{tabular}{|c|c|c|}
\hline $\begin{array}{l}\text { No. } \\
\left(h_{i}(x)\right)\end{array}$ & Coefficients & Injected Boolean Function \\
\hline $\mathbf{0}$ & - & $\begin{array}{l}x_{1}+x_{4}+x_{0} x_{3}+x_{2} x_{3}+x_{3} x_{4}+x_{0} x_{1} x_{2}+x_{0} x_{2} x_{3} \\
+x_{0} x_{2} x_{4}+x_{1} x_{2} x_{4}+x_{2} x_{3} x_{4}\end{array}$ \\
\hline 1 & $x_{0}$ & $\begin{array}{l}x_{1}+x_{4}+x_{3}+x_{1} x_{2}+x_{2} x_{4}+x_{3} x_{4}+x_{1} x_{2} x_{4}+ \\
x_{2} x_{3} x_{4}\end{array}$ \\
\hline 2 & $x_{1}$ & $\begin{array}{l}1+x_{4}+x_{0} x_{2}+x_{0} x_{3}+x_{2} x_{3}+x_{2} x_{4}+x_{3} x_{4}+ \\
x_{0} x_{2} x_{3}+x_{0} x_{2} x_{4}+x_{2} x_{3} x_{4}\end{array}$ \\
\hline 3 & $x_{2}$ & $x_{1}+x_{3}+x_{4}+x_{0} x_{1}+x_{0} x_{4}+x_{1} x_{4}$ \\
\hline 4 & $x_{3}$ & $\begin{array}{l}x_{0}+x_{1}+x_{2}+x_{0} x_{2}+x_{2} x_{4}+x_{0} x_{1} x_{2}+x_{0} x_{2} x_{4} \\
+x_{1} x_{2} x_{4}\end{array}$ \\
\hline 5 & $x_{4}$ & $x_{1}+x_{3}+x_{0} x_{2}+x_{0} x_{3}+x_{1} x_{2}+x_{0} x_{1} x_{2}+x_{0} x_{2} x_{3}$ \\
\hline 6 & $x_{0} x_{1}$ & $\begin{array}{l}x_{1}+x_{2}+x_{4}+x_{0} x_{3}+x_{2} x_{3}+x_{3} x_{4}+x_{0} x_{2} x_{3}+ \\
x_{0} x_{2} x_{4}+x_{1} x_{2} x_{4}+x_{2} x_{3} x_{4}\end{array}$ \\
\hline 7 & $x_{0} X_{2}$ & $x_{3}+x_{0} x_{3}+x_{2} x_{3}+x_{3} x_{4}+x_{1} x_{2} x_{4}+x_{2} x_{3} x_{4}$ \\
\hline 8 & $x_{0} x_{3}$ & $\begin{array}{l}x_{1}+x_{2}+x_{4}+1+x_{2} x_{3}+x_{3} x_{4}+x_{0} x_{1} x_{2}+ \\
x_{0} x_{2} x_{4}+x_{1} x_{2} x_{4}+x_{2} x_{3} x_{4}\end{array}$ \\
\hline 9 & $x_{0} x_{4}$ & $\begin{array}{l}x_{1}+x_{2}+x_{4}+x_{0} x_{3}+x_{2} x_{3}+x_{3} x_{4}+x_{0} x_{1} x_{2} \\
+x_{0} x_{2} x_{3}+x_{1} x_{2} x_{4}+x_{2} x_{3} x_{4}\end{array}$ \\
\hline 10 & $x_{1} x_{2}$ & $\begin{array}{l}x_{0}+x_{1}+x_{0} x_{3}+x_{2} x_{3}+x_{3} x_{4}+x_{0} x_{2} x_{3}+x_{0} x_{2} x_{4} \\
+x_{2} X_{3} X_{4}\end{array}$ \\
\hline 11 & $x_{1} x_{4}$ & $\begin{array}{l}x_{1}+x_{2}+x_{4}+x_{0} x_{3}+x_{2} x_{3}+x_{3} x_{4}+x_{0} x_{1} x_{2} \\
+x_{0} x_{2} x_{3}+x_{0} x_{2} x_{4}+x_{2} x_{3} x_{4}\end{array}$ \\
\hline 12 & $x_{2} x_{3}$ & $\begin{array}{l}1+x_{0}+x_{1}+x_{0} x_{3}+x_{3} x_{4}+x_{0} x_{1} x_{2}+x_{0} x_{2} x_{4} \\
+x_{1} x_{2} X_{4}\end{array}$ \\
\hline 13 & $x_{2} X_{4}$ & $\begin{array}{l}x_{1}+x_{4}+x_{0} x_{3}+x_{2} x_{3}+x_{3} x_{4}+x_{0} x_{1} x_{2}+x_{0} x_{2} x_{3} \\
+x_{0} x_{2} X_{4}+x_{1} x_{2} x_{4}+x_{3}\end{array}$ \\
\hline 14 & $x_{3} x_{4}$ & $\begin{array}{l}x_{1}+x_{2}+x_{4}+x_{0} x_{3}+x_{2} x_{3}+x_{0} x_{1} x_{2}+x_{0} x_{2} x_{3}+ \\
x_{0} x_{2} x_{4}+x_{1} x_{2} x_{4}\end{array}$ \\
\hline 15 & $x_{0} x_{1} x_{2}$ & $\begin{array}{l}1+x_{1}+x_{4}+x_{0} x_{3}+x_{2} x_{3}+x_{3} x_{4}+x_{0} x_{2} x_{3}+ \\
x_{0} x_{2} x_{4}+x_{1} x_{2} x_{4}+x_{2} x_{3} x_{4}\end{array}$ \\
\hline 16 & $x_{0} x_{2} X_{3}$ & $\begin{array}{l}1+x_{1}+x_{4}+x_{0} x_{3}+x_{2} x_{3}+x_{3} x_{4}+x_{0} x_{1} x_{2}+ \\
x_{0} x_{2} x_{4}+x_{1} x_{2} x_{4}+x_{2} x_{3} x_{4}\end{array}$ \\
\hline 17 & $x_{0} X_{2} X_{4}$ & $\begin{array}{l}1+x_{1}+x_{4}+x_{0} x_{3}+x_{2} x_{3}+x_{3} x_{4}+x_{0} x_{1} x_{2} \\
+x_{0} x_{2} x_{3}+x_{1} x_{2} x_{4}+x_{2} x_{3} x_{4}\end{array}$ \\
\hline 18 & $x_{1} X_{2} X_{4}$ & $\begin{array}{l}1+x_{1}+x_{4}+x_{0} x_{3}+x_{2} x_{3}+x_{3} x_{4}+x_{0} x_{1} x_{2} \\
+x_{0} x_{2} x_{3}+x_{0} x_{2} x_{4}+x_{2} x_{3} x_{4}\end{array}$ \\
\hline 19 & $X_{2} X_{3} X_{4}$ & $\begin{array}{l}1+x_{1}+x_{4}+x_{0} x_{3}+x_{2} x_{3}+x_{3} x_{4}+x_{0} x_{1} x_{2} \\
+x_{0} x_{2} x_{3}+x_{0} x_{2} x_{4}+x_{1} x_{2} x_{4}\end{array}$ \\
\hline
\end{tabular}

Table 1: List of Injected Grain v0’s Boolean function (above).

\begin{tabular}{|c|l|}
\hline$\left(\boldsymbol{h}_{\boldsymbol{i}}(\boldsymbol{x})\right)$ & \multicolumn{1}{|c|}{$\left(\boldsymbol{h}_{\boldsymbol{i}}(\boldsymbol{x})\right)$ in vector } \\
\hline $\mathbf{0}$ & $\begin{array}{l}(0,1,0,0,1,0,0,1,0,0,0,0,1,0,1,1,0,0,1,1,0,0,1,0, \\
1,0,0,0,0,0,0)\end{array}$ \\
\hline 1 & $\begin{array}{l}(0,1,0,1,1,0,0,0,0,1,0,0,0,1,1,0,0,0,0,0,0,0,1,0, \\
1,0,0,0,0,0,0)\end{array}$ \\
\hline 2 & $\begin{array}{l}(1,0,0,0,0,1,0,1,1,0,0,0,0,1,1,1,0,0,0,1,1,0,0,0, \\
0,1,0,0,0,0,0,0)\end{array}$ \\
\hline 3 & $\begin{array}{l}(1,0,0,0,0,1,0,1,1,0,0,0,0,1,1,1,0,0,0,1,1,0,0,0, \\
0,1,0,0,0,0,0,0)\end{array}$ \\
\hline
\end{tabular}




\begin{tabular}{|c|c|}
\hline 4 & $\begin{array}{l}(0,1,0,1,1,1,0,0,1,0,0,1,0,0,0,0,0,0,0,0,0,0,0,0, \\
0,0,0,0,0,0,0)\end{array}$ \\
\hline 5 & $\begin{array}{l}(1,1,1,0,0,0,1,0,0,0,0,0,0,1,0,1,0,0,0,1,0,0,1,0, \\
0,0,0,0,0,0,0)\end{array}$ \\
\hline 6 & $\begin{array}{l}(0,1,1,0,1,0,0,1,0,0,0,0,1,0,1,0,0,0,1,1,0,0,1,0, \\
1,0,0,0,0,0,0)\end{array}$ \\
\hline 7 & $\begin{array}{l}(0,0,0,1,0,0,0,1,0,0,0,0,1,0,1,0,0,0,0,0,0,0,1,0, \\
1,0,0,0,0,0,0)\end{array}$ \\
\hline 8 & $\begin{array}{l}(1,0,1,1,0,1,0,0,0,0,0,0,0,1,0,1,1,0,0,0,1,0,0,1, \\
0,1,0,0,0,0,0,0)\end{array}$ \\
\hline 9 & $\begin{array}{l}(0,1,1,0,1,0,0,1,0,0,0,0,1,0,1,1,0,0,1,0,0,0,1,0, \\
1,0,0,0,0,0,0)\end{array}$ \\
\hline 10 & $\begin{array}{l}(1,1,0,0,0,0,0,1,0,0,0,0,1,0,1,0,0,0,1,1,0,0,0,0, \\
1,0,0,0,0,0,0)\end{array}$ \\
\hline 11 & $\begin{array}{l}(0,1,1,0,1,0,0,1,0,0,0,0,1,0,1,1,0,0,1,1,0,0,0,0, \\
1,0,0,0,0,0,0)\end{array}$ \\
\hline 12 & $\begin{array}{l}(1,1,1,0,0,0,0,0,1,0,0,0,0,0,0,1,1,0,0,0,1,0,0,1, \\
0,0,0,0,0,0,0,0)\end{array}$ \\
\hline 13 & $\begin{array}{l}(0,1,0,1,1,0,0,1,0,0,0,0,1,0,1,1,0,0,1,1,0,0,1,0, \\
0,0,0,0,0,0,0)\end{array}$ \\
\hline 14 & $\begin{array}{l}(0,1,1,0,1,0,0,1,0,0,0,0,1,0,0,1,0,0,1,1,0,0,1,0, \\
0,0,0,0,0,0,0)\end{array}$ \\
\hline 15 & $\begin{array}{l}(1,0,1,0,0,1,0,0,1,0,0,0,0,1,0,1,0,0,0,1,1,0,0,1, \\
0,1,0,0,0,0,0,0)\end{array}$ \\
\hline 16 & $\begin{array}{l}(1,0,1,0,0,1,0,0,1,0,0,0,0,1,0,1,1,0,0,0,1,0,0,1, \\
0,1,0,0,0,0,0,0)\end{array}$ \\
\hline 17 & $\begin{array}{l}(1,0,1,0,0,1,0,0,1,0,0,0,0,1,0,1,1,0,0,1,0,0,0,1, \\
0,1,0,0,0,0,0,0)\end{array}$ \\
\hline 18 & $\begin{array}{l}(1,0,1,0,0,1,0,0,1,0,0,0,0,1,0,1,1,0,0,1,1,0,0,0, \\
0,1,0,0,0,0,0,0)\end{array}$ \\
\hline 19 & $\begin{array}{l}(1,0,1,0,0,1,0,0,1,0,0,0,0,1,0,1,1,0,0,1,1,0,0,1, \\
0,0,0,0,0,0,0,0)\end{array}$ \\
\hline
\end{tabular}

Table 2: List of Injected Grain v0’s in vector (above).

From Table 2, value of $\left(h_{i}(x)\right)$ in vector will be used to compute value of $\mathrm{C}=\left\{p \bullet h \mid p \in \mathrm{A}_{2}\right\}$. We construct matrix $M_{d}(h)$ by output of $\mathrm{C}$ for each injected Boolean function and we get [15x31] matrices. Table 3 show output of $M_{d}(h)$

\begin{tabular}{|c|c|}
\hline$h_{i}(x)$ & $M_{d}\left(h_{i}\right)$ \\
\hline 0 & $\begin{array}{lllllllllllllllllllllllllllllll}0 & 0 & 0 & 0 & 0 & 1 & 0 & 1 & 1 & 0 & 0 & 0 & 0 & 0 & 0 & 1 & 0 & 0 & 0 & 1 & 1 & 0 & 0 & 0 & 0 & 0 & 1 & 0 & 1 & 0 & 0 \\
0 & 1 & 0 & 0 & 0 & 0 & 0 & 0 & 0 & 0 & 0 & 1 & 0 & 0 & 0 & 1 & 1 & 0 & 0 & 0 & 0 & 1 & 1 & 1 & 0 & 1 & 1 & 0 & 0 & 1 & 0 \\
0 & 0 & 0 & 0 & 0 & 0 & 0 & 0 & 0 & 1 & 0 & 0 & 1 & 1 & 0 & 1 & 0 & 0 & 0 & 1 & 0 & 0 & 1 & 0 & 0 & 0 & 0 & 0 & 0 & 0 & 0 \\
0 & 0 & 0 & 0 & 0 & 0 & 0 & 1 & 0 & 0 & 1 & 0 & 1 & 0 & 0 & 0 & 0 & 0 & 1 & 0 & 0 & 0 & 0 & 0 & 1 & 1 & 0 & 0 & 1 & 1 & 0 \\
0 & 0 & 0 & 0 & 1 & 0 & 0 & 0 & 0 & 0 & 0 & 1 & 0 & 0 & 1 & 0 & 0 & 0 & 0 & 1 & 1 & 0 & 1 & 0 & 0 & 0 & 1 & 0 & 1 & 0 & 0 \\
0 & 0 & 0 & 0 & 0 & 1 & 0 & 0 & 0 & 0 & 0 & 0 & 0 & 0 & 0 & 1 & 1 & 1 & 0 & 0 & 0 & 0 & 0 & 0 & 0 & 0 & 0 & 1 & 0 & 0 & 1 \\
0 & 0 & 0 & 0 & 0 & 0 & 0 & 0 & 0 & 0 & 0 & 0 & 0 & 0 & 0 & 0 & 0 & 0 & 1 & 0 & 0 & 0 & 0 & 0 & 0 & 0 & 1 & 0 & 0 & 0 & 0 \\
0 & 0 & 0 & 0 & 0 & 0 & 0 & 1 & 0 & 0 & 0 & 0 & 0 & 0 & 0 & 0 & 1 & 0 & 0 & 0 & 0 & 0 & 0 & 0 & 0 & 1 & 0 & 0 & 0 & 0 & 1 \\
0 & 0 & 0 & 0 & 0 & 0 & 0 & 0 & 1 & 0 & 0 & 0 & 0 & 0 & 0 & 0 & 0 & 1 & 0 & 1 & 0 & 0 & 0 & 0 & 0 & 0 & 0 & 0 & 1 & 0 & 0 \\
0 & 0 & 0 & 0 & 0 & 0 & 0 & 0 & 0 & 1 & 0 & 0 & 0 & 0 & 0 & 1 & 0 & 0 & 0 & 0 & 0 & 1 & 0 & 0 & 0 & 0 & 1 & 0 & 0 & 0 & 0 \\
0 & 0 & 0 & 0 & 0 & 0 & 0 & 0 & 0 & 0 & 1 & 0 & 0 & 0 & 0 & 0 & 1 & 0 & 0 & 0 & 0 & 1 & 0 & 0 & 0 & 0 & 0 & 0 & 0 & 0 & 1 \\
0 & 0 & 0 & 0 & 0 & 0 & 0 & 0 & 0 & 0 & 0 & 0 & 0 & 0 & 0 & 0 & 0 & 0 & 0 & 0 & 0 & 0 & 1 & 1 & 0 & 0 & 0 & 1 & 0 & 0 & 1 \\
0 & 0 & 0 & 0 & 0 & 0 & 0 & 0 & 0 & 0 & 0 & 0 & 1 & 0 & 0 & 0 & 0 & 0 & 0 & 0 & 0 & 1 & 0 & 0 & 1 & 1 & 0 & 0 & 1 & 1 & 0 \\
0 & 0 & 0 & 0 & 0 & 0 & 0 & 0 & 0 & 0 & 0 & 0 & 0 & 1 & 0 & 0 & 0 & 0 & 0 & 1 & 0 & 0 & 0 & 0 & 1 & 0 & 1 & 0 & 0 & 0 & 0 \\
0 & 0 & 0 & 0 & 0 & 0 & 0 & 0 & 0 & 0 & 0 & 0 & 0 & 0 & 0 & 0 & 0 & 0 & 0 & 0 & 1 & 0 & 0 & 1 & 0 & 0 & 0 & 0 & 0 & 1 & 1\end{array}$ \\
\hline 1 & 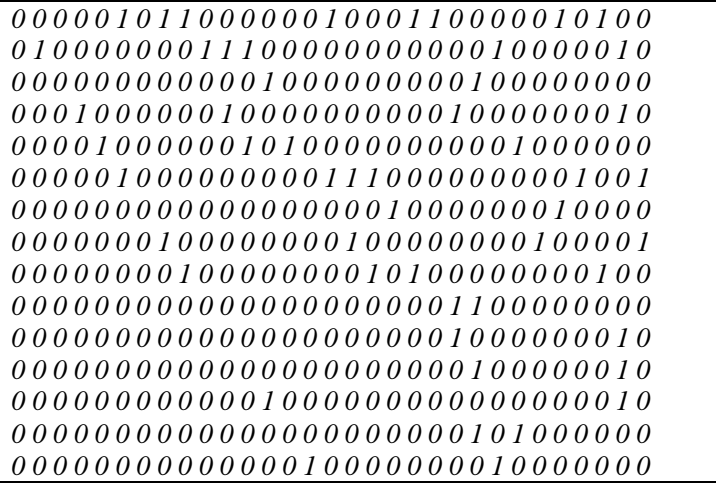 \\
\hline 2 & 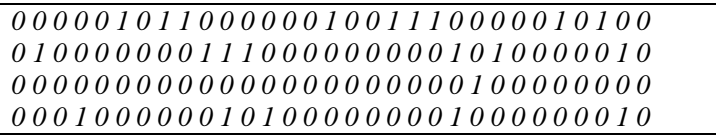 \\
\hline
\end{tabular}

\begin{tabular}{|c|c|}
\hline & 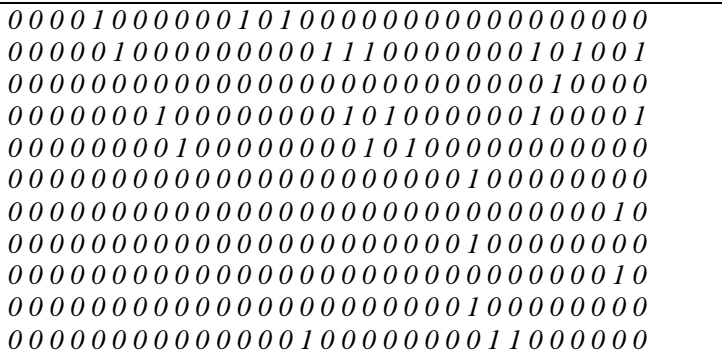 \\
\hline 3 & 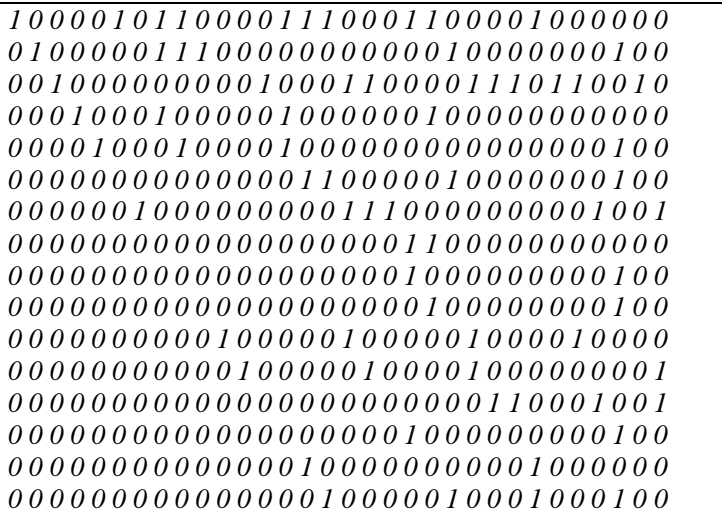 \\
\hline 4 & $\begin{array}{lllllllllllllllllllllllllllllll}0 & 0 & 0 & 0 & 0 & 0 & 0 & 1 & 0 & 0 & 0 & 0 & 0 & 0 & 0 & 0 & 0 & 1 & 0 & 0 & 0 & 0 & 0 & 0 & 0 & 0 & 0 & 0 & 0 & 0 & 0 \\
0 & 1 & 0 & 0 & 0 & 1 & 0 & 0 & 0 & 0 & 1 & 0 & 0 & 0 & 0 & 0 & 0 & 1 & 0 & 0 & 0 & 0 & 0 & 0 & 0 & 0 & 0 & 0 & 0 & 0 & 0 \\
0 & 0 & 0 & 0 & 0 & 0 & 0 & 0 & 0 & 1 & 0 & 0 & 1 & 1 & 0 & 1 & 0 & 0 & 0 & 1 & 0 & 0 & 1 & 0 & 0 & 0 & 0 & 0 & 0 & 0 & 0 \\
0 & 0 & 0 & 1 & 0 & 0 & 0 & 0 & 0 & 0 & 1 & 0 & 0 & 0 & 1 & 0 & 1 & 0 & 0 & 0 & 1 & 0 & 0 & 1 & 0 & 0 & 0 & 0 & 0 & 0 & 0 \\
0 & 0 & 0 & 0 & 1 & 0 & 0 & 0 & 1 & 0 & 0 & 0 & 0 & 0 & 1 & 0 & 0 & 1 & 0 & 0 & 0 & 0 & 0 & 0 & 0 & 0 & 0 & 0 & 0 & 0 & 0 \\
0 & 0 & 0 & 0 & 0 & 0 & 0 & 0 & 0 & 0 & 0 & 0 & 0 & 0 & 0 & 0 & 1 & 1 & 0 & 0 & 0 & 0 & 0 & 0 & 0 & 0 & 0 & 0 & 0 & 0 & 0 \\
0 & 0 & 0 & 0 & 0 & 0 & 0 & 0 & 0 & 0 & 0 & 0 & 0 & 0 & 0 & 0 & 0 & 0 & 1 & 0 & 0 & 0 & 0 & 0 & 0 & 0 & 1 & 0 & 0 & 0 & 0 \\
0 & 0 & 0 & 0 & 0 & 0 & 0 & 1 & 0 & 0 & 0 & 0 & 0 & 0 & 0 & 0 & 0 & 0 & 0 & 0 & 0 & 0 & 0 & 0 & 0 & 0 & 0 & 1 & 0 & 0 & 0 \\
0 & 0 & 0 & 0 & 0 & 0 & 0 & 0 & 0 & 0 & 0 & 0 & 0 & 0 & 0 & 0 & 0 & 1 & 0 & 0 & 1 & 0 & 0 & 0 & 0 & 0 & 0 & 0 & 0 & 0 & 0 \\
0 & 0 & 0 & 0 & 0 & 0 & 0 & 0 & 0 & 1 & 0 & 0 & 0 & 0 & 0 & 1 & 0 & 0 & 0 & 0 & 0 & 1 & 0 & 0 & 0 & 0 & 1 & 0 & 0 & 0 & 0 \\
0 & 0 & 0 & 0 & 0 & 0 & 0 & 0 & 0 & 0 & 0 & 0 & 0 & 0 & 0 & 0 & 1 & 0 & 0 & 0 & 0 & 0 & 0 & 0 & 0 & 0 & 0 & 1 & 0 & 0 & 0 \\
0 & 0 & 0 & 0 & 0 & 0 & 0 & 0 & 0 & 0 & 0 & 1 & 0 & 0 & 0 & 0 & 0 & 0 & 0 & 0 & 0 & 0 & 0 & 1 & 0 & 0 & 0 & 0 & 0 & 0 & 0 \\
0 & 0 & 0 & 0 & 0 & 0 & 0 & 0 & 0 & 0 & 0 & 0 & 1 & 0 & 0 & 0 & 0 & 0 & 0 & 0 & 0 & 1 & 0 & 0 & 1 & 1 & 0 & 0 & 1 & 1 & 0 \\
0 & 0 & 0 & 0 & 0 & 0 & 0 & 0 & 0 & 0 & 0 & 0 & 0 & 1 & 0 & 0 & 0 & 0 & 0 & 1 & 0 & 0 & 0 & 0 & 1 & 0 & 1 & 0 & 0 & 0 & 0 \\
0 & 0 & 0 & 0 & 0 & 0 & 0 & 0 & 0 & 0 & 0 & 0 & 0 & 0 & 0 & 0 & 0 & 0 & 0 & 0 & 1 & 0 & 0 & 0 & 0 & 0 & 0 & 1 & 0 & 0 & 0\end{array}$ \\
\hline 5 & 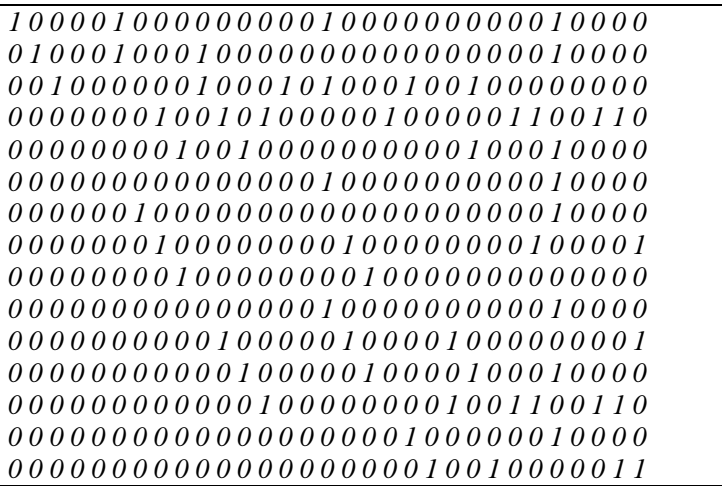 \\
\hline 6 & 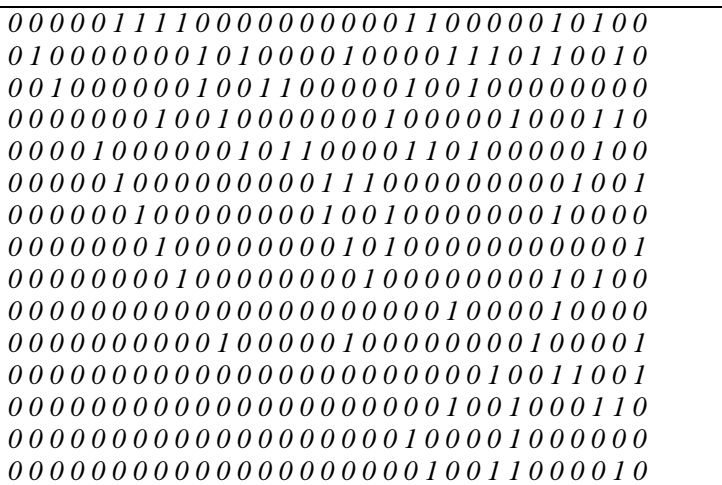 \\
\hline 7 & 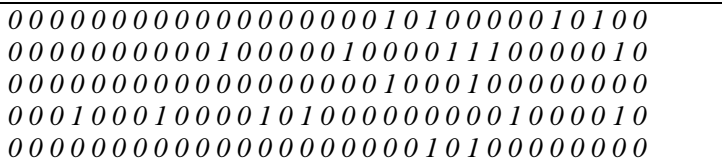 \\
\hline
\end{tabular}




\begin{tabular}{|c|c|}
\hline & 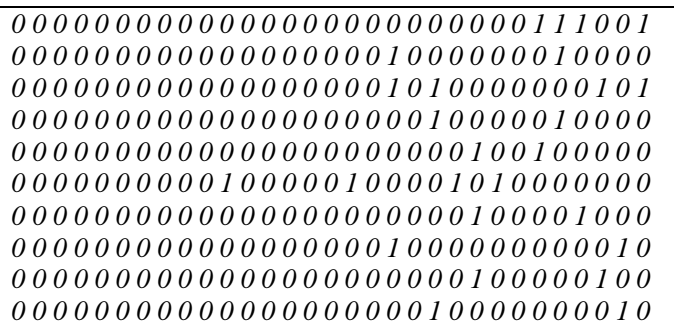 \\
\hline 8 & 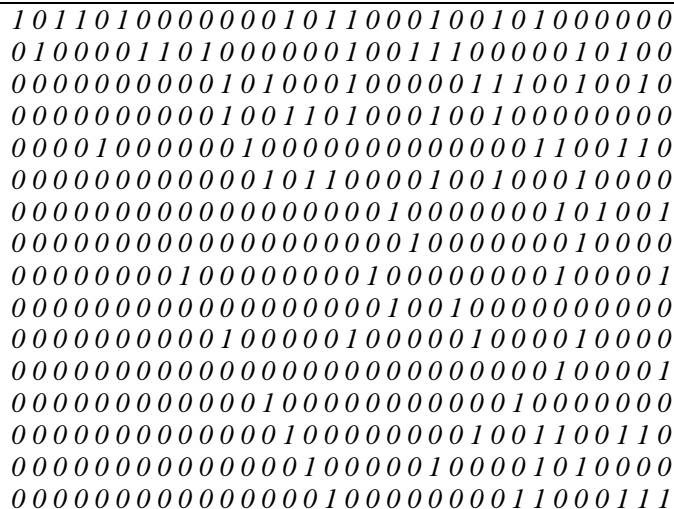 \\
\hline 9 & 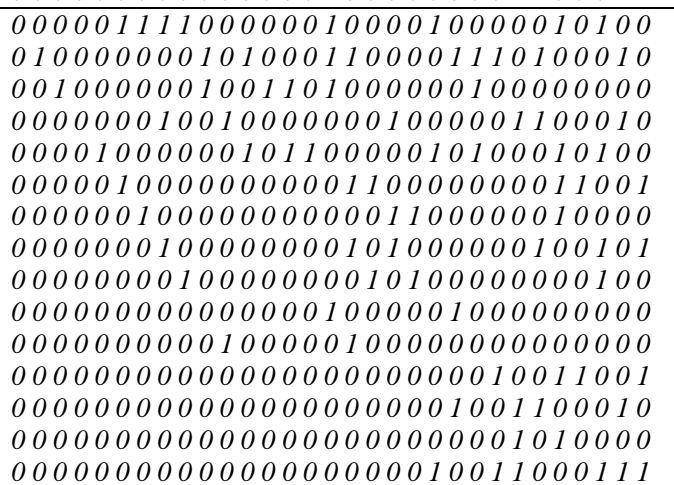 \\
\hline 10 & 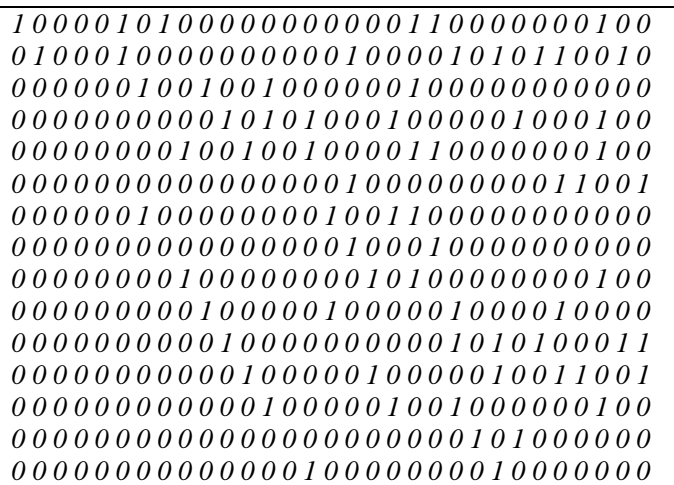 \\
\hline 11 & 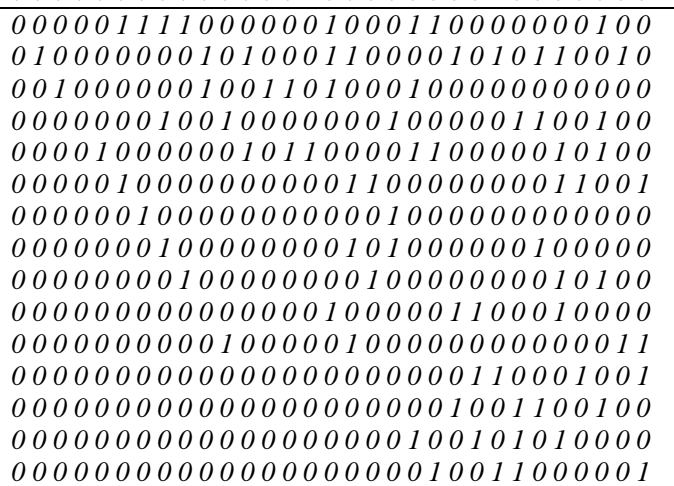 \\
\hline 12 & 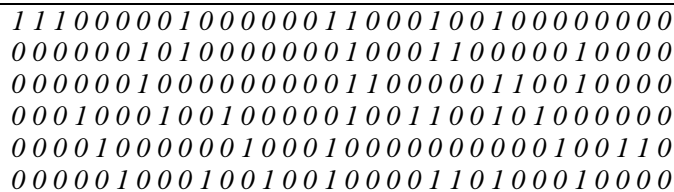 \\
\hline
\end{tabular}

\begin{tabular}{|c|c|}
\hline & 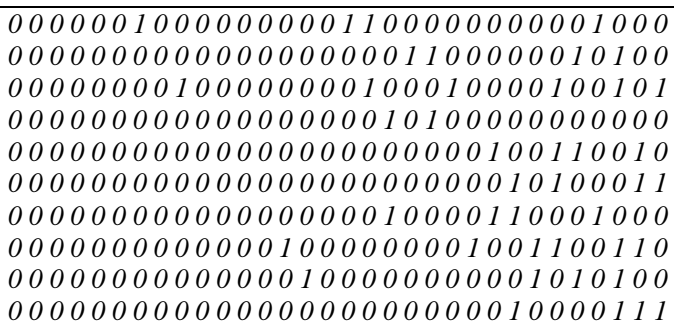 \\
\hline 13 & 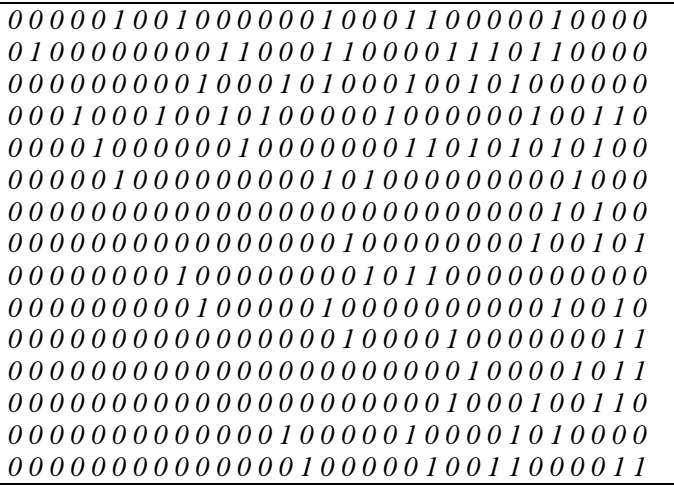 \\
\hline 14 & 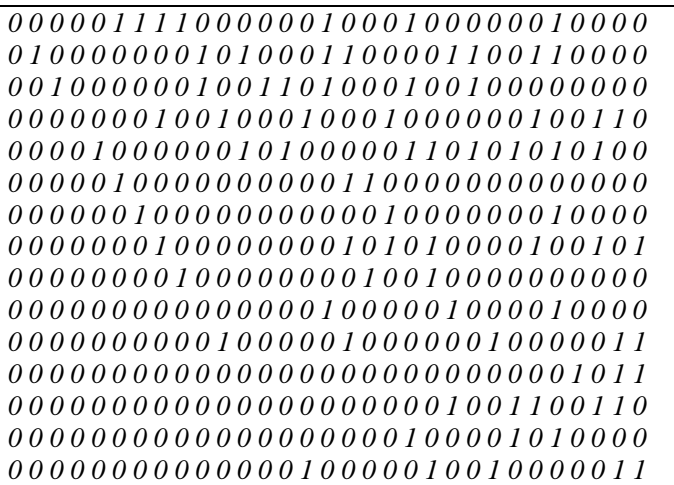 \\
\hline 15 & 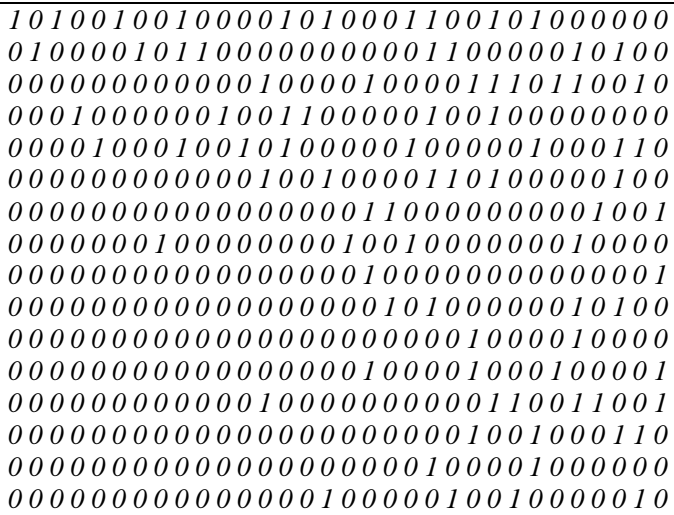 \\
\hline 16 & 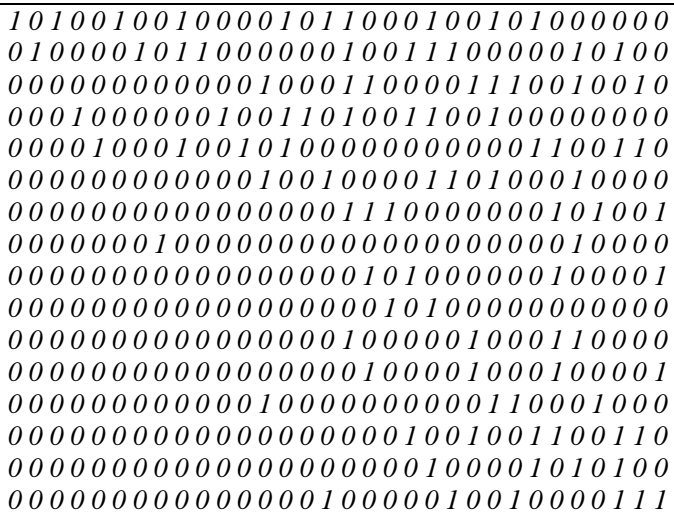 \\
\hline 17 & 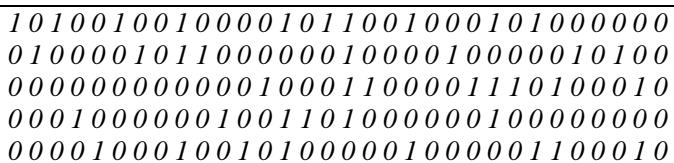 \\
\hline
\end{tabular}




\begin{tabular}{|c|c|}
\hline & 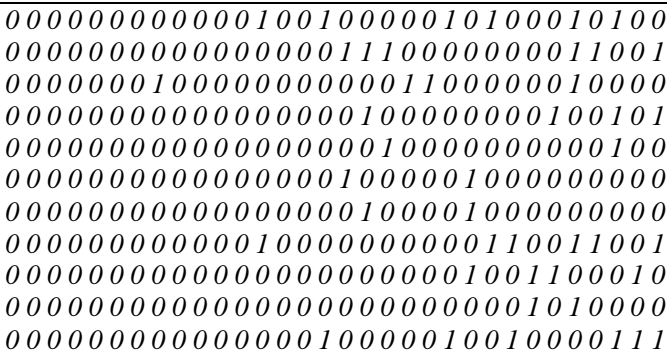 \\
\hline 18 & 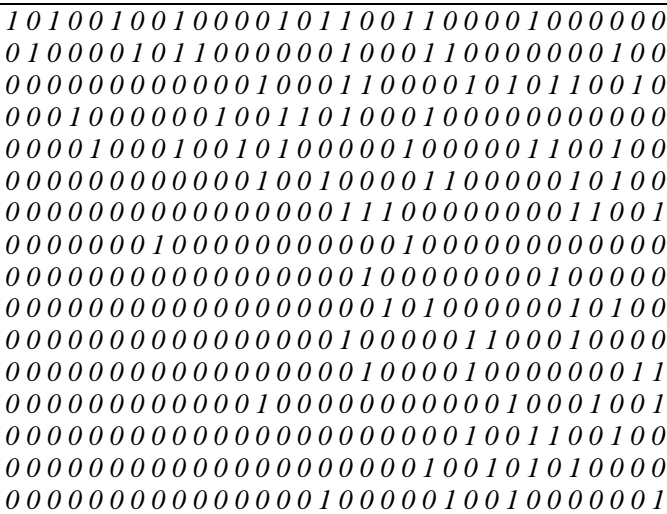 \\
\hline 19 & 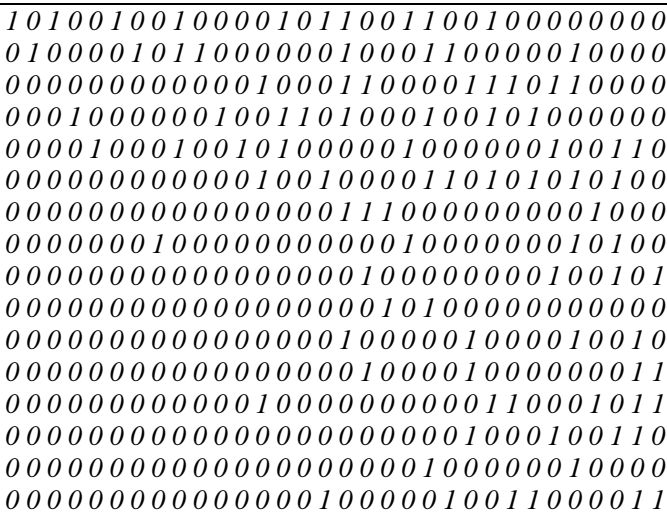 \\
\hline
\end{tabular}

Table 3: Output of $M_{d}(h)$ (above).

From Table 3, we convert $M_{d}(h)$ into row ladder matrix - $M_{d}(h) *$ using Gaussian elimination. For this purpose, we are using Maple 18 (with command: ReducedRowEchelonForm(x)). As for the output of $M_{d}(h)^{*}$ show as in Table 4.

\begin{tabular}{|c|c|}
\hline$h_{i}(x)$ & $M_{d}\left(h_{i}\right)^{*}$ \\
\hline 0 & 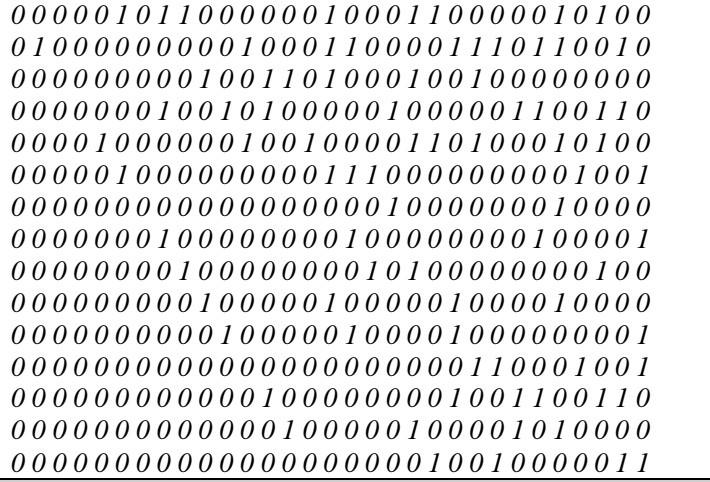 \\
\hline 1 & 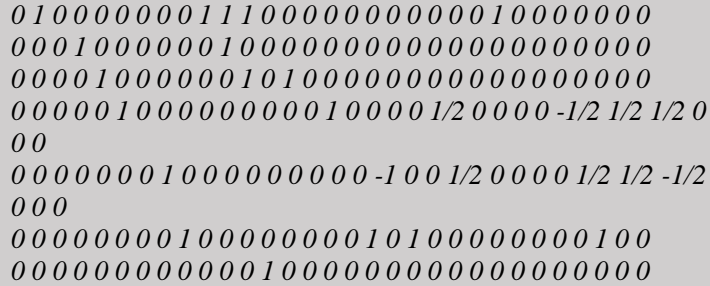 \\
\hline
\end{tabular}

\begin{tabular}{|c|c|}
\hline & 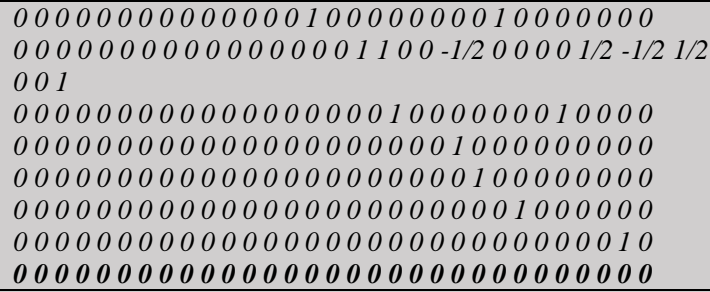 \\
\hline 2 & 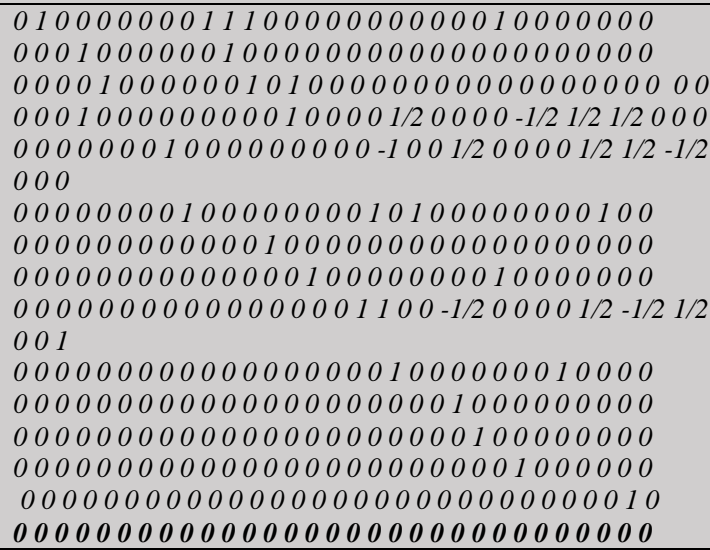 \\
\hline 3 & 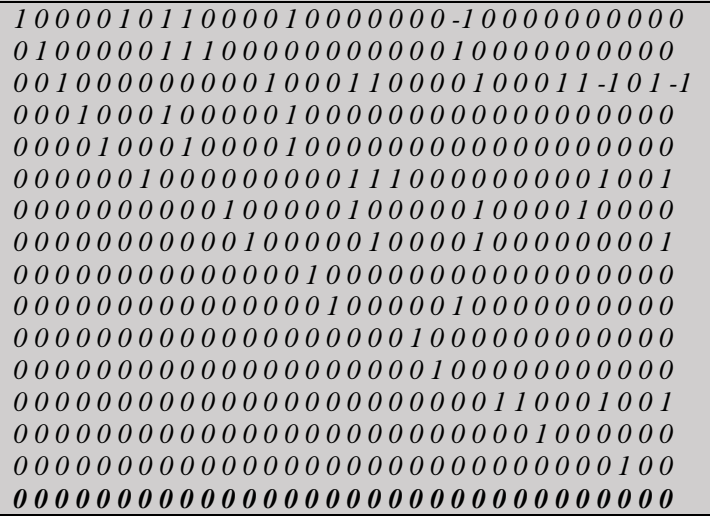 \\
\hline 4 & 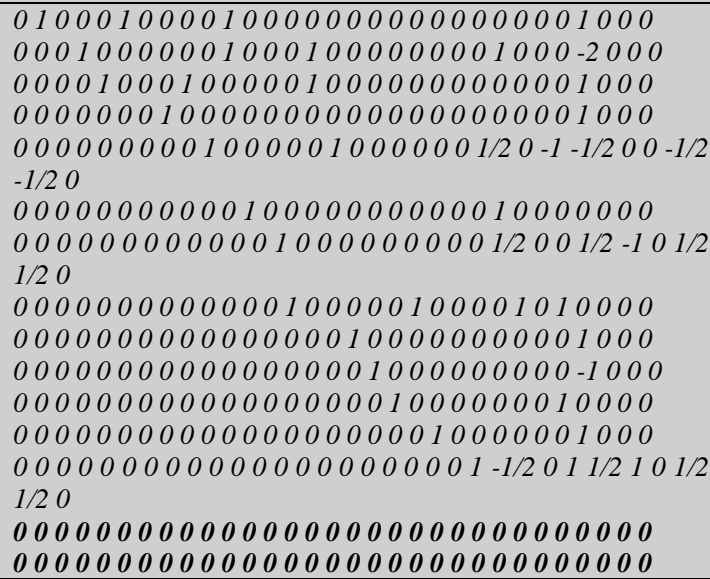 \\
\hline 5 & 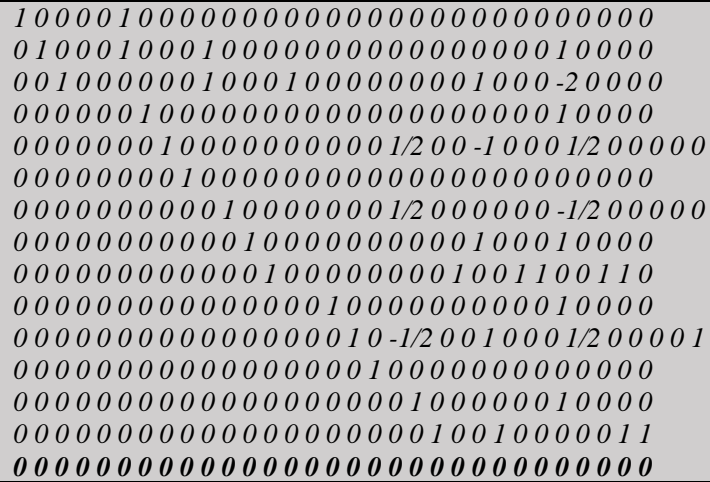 \\
\hline & $01000000010100000000001001 / 2-1 / 2-101$ \\
\hline
\end{tabular}




\begin{tabular}{|c|c|}
\hline & $\begin{array}{l}00100000010011000000001000-10110 \\
00001000000101100000001000-11311 \\
000000100000000000000-100000000-1111 / 2 \\
1 / 2 \\
000000010000000000-100000001 / 2-1 / 201 \\
1 / 21 / 2 \\
000000001000000000001000000-1 / 21 / 20000 \\
00000000010000000010000000010100 \\
0000000000010000000000000001 / 21 / 20000 \\
000000000000000001011000000-1 / 23 / 20-1 \text { - } \\
1 / 2-1 / 2 \\
0000000000000000000010000000001 / 2-1 / 20001 \\
000000000000000000000100000010-1-10 \\
0000000000000000000000001000000-1-10-1 \\
000000000000000000000001000010000 \\
00000000000000000000000000010011001 \\
000000000000000000000000000010-10110\end{array}$ \\
\hline 7 & 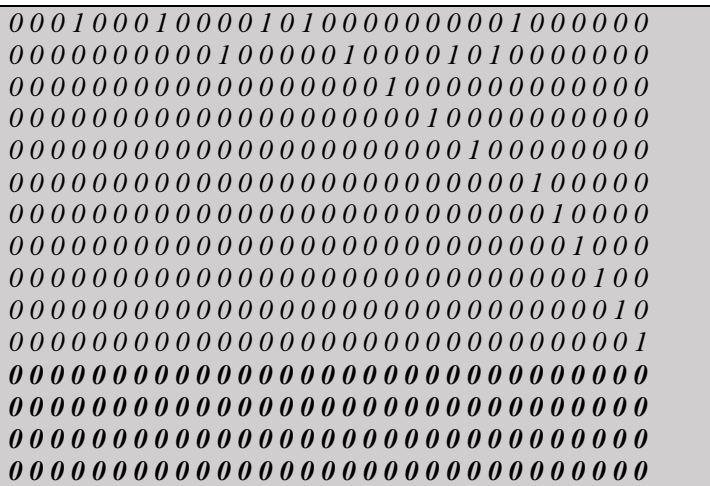 \\
\hline 8 & $\begin{array}{l}10110100000000001000100001010-1-10 \\
01000011010000001000100000001100 \\
0000100000010000000000000100011-1 \\
00000000100000000100000000000000 \\
0000000000100000100000000-1000-1 / 2-1 \\
1 / 2 \\
00000000000001000000000000-1000-1 / 2-1 \\
-1 / 2 \\
0000000000000010000000000000-101 / 20- \\
1 / 2 \\
000000000000000100000100001010000 \\
000000000000000100000000000001 / 20 \\
1 / 2 \\
000000000000000000010000000001000 \\
00000000000000000001000000010000 \\
00000000000000000000001000000-1000 \\
0000000000000000000000010010101 / 21- \\
1 / 2 \\
0000000000000000000000000100000010 \\
000000000000000000000000110001 / 21 \\
1 / 2 \\
00000000000000000000000000010000\end{array}$ \\
\hline 9 & 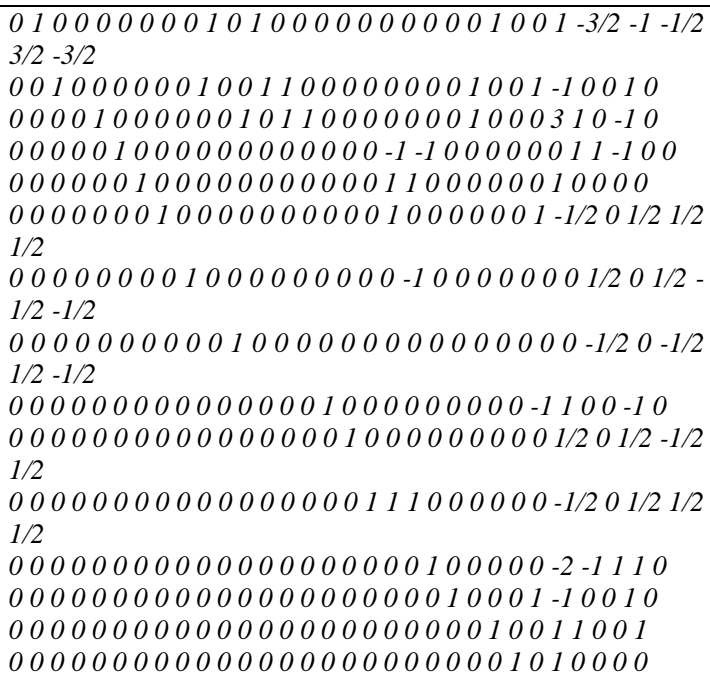 \\
\hline & 1000010100000000000100000011101 \\
\hline
\end{tabular}

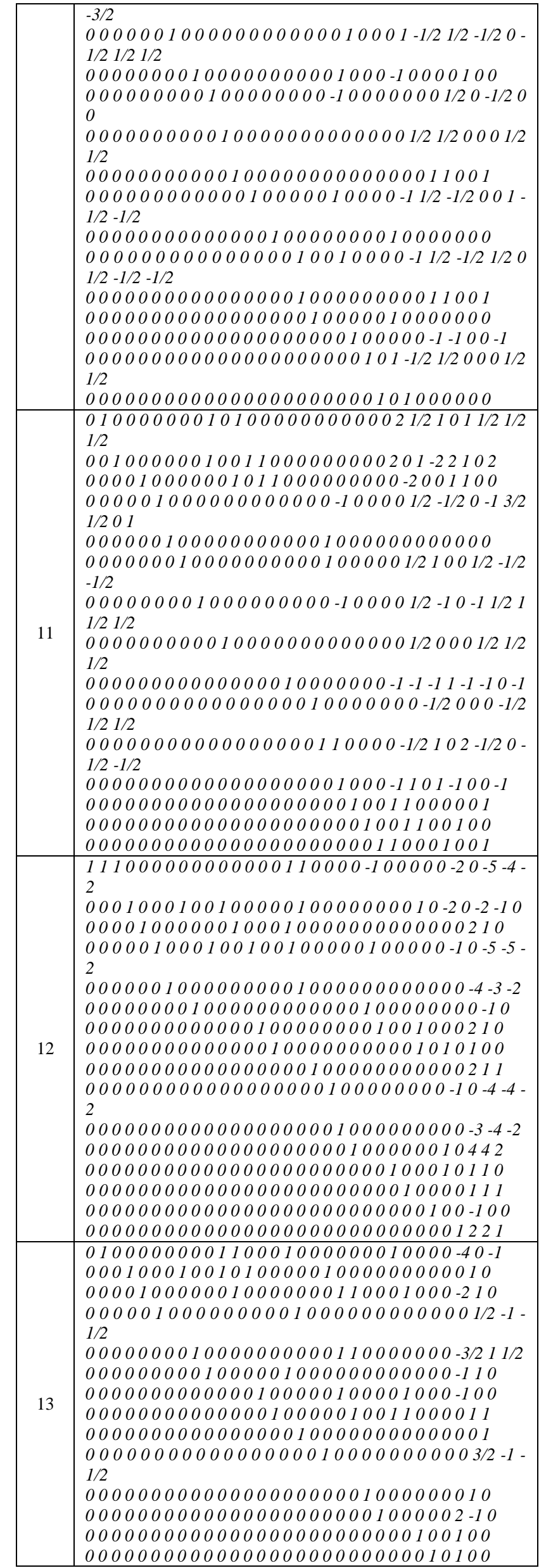


$0000000000000000000000000001-221$

$01000000010100000000-101-101000-1 / 2$ $3 / 200100000010011000000001001-2011$ 000001000000101000000101000001000 $00001000000000000-10-100000-1001 / 2$ $1 / 20000001000000000001000000010000$ $00000001000000000010000-101001-1 / 2$ $1 / 2000000001000000000-10100100-1001$ $100000000001000000000-1000000001 / 2$ $1 / 20000000000000010000010010000011$ $000000000000000100000000-1-110-1-10$ $000000000000000010001001000001 / 23 / 2$ $00000000000000000110000-100100-1-10$ 000000000000000000100001010000000 000000000000000000100110011000000 00000000000000000000001011

$101001001000010000010-101000100-1 / 2$ $1 / 2$

$0100001011000000000001000000001-2$ $000100000010011000000001000-1001-1$ 00001000100101000001000000010000 00000001000000001001000000010000 $0000000000001000000000010000001 / 2$ $3 / 2$

15 $000000000000000100000100000-1001 / 2$. $1 / 2$

00000000000000000100000000000001 $0000000000000000001000000000001-2$ $000000000000000000001000000100-11$ 00000000000000000000001000010000 $0000000000000000000000001001001 / 2$ $1 / 2$

$000000000000000000000000010-1001-1$ $000000000000000000000000001-10000$ $000000000000000000000000000010-1$

$101001001000010000000-101000-25 / 12$ $3 / 2-11 / 12-1 / 6$

$010000101100000000000100000-14 / 31$ $1 / 32 / 3$

$000100000010011000000001000-24 / 30$ $1 / 32 / 3$

$0000100010010100000000000000-4 / 304 / 3$ $-2 / 3$

00000001000000000000000000010000 $00000000000010000000000100003 / 4-1 / 2$ $1 / 4-1 / 2$

$0000000000000001000001000000-1 / 41 / 2$ $3 / 41 / 2$

$0000000000000000100000000001-1 / 601 / 6$ $-1 / 3$

$160000000000000000010000000000-1 / 601 / 6$ $2 / 3$

$000000000000000000100000000-11 / 201 / 2$ 0 $0000000000000000000100000000-2 / 302 / 3$ $-1 / 3$

$0000000000000000000010000001-1 / 20$ $1 / 20$

$0000000000000000000000100000-2 / 302 / 3$ $-1 / 3$

$00000000000000000000000010001 / 41 / 2$ $1 / 41 / 2$

$00000000000000000000000001001 / 211 / 2$ 0

$00000000000000000000000000105 / 60-5 / 6$ $2 / 3$

$101001001000010000010-101000004-7 / 2$ $5 / 2$

01000010110000000000010000000 -13/2 $3 / 2$

$000100000010011000000001000001-1 / 2$ $1 / 2$

$000010001001010000010000000001-1 / 2$ $1 / 2$

$00000001000000000001100000000-32-2$ $00000000000010000000000100000-21-2$ 00000000000000010000010000000010

\begin{tabular}{|c|c|}
\hline & $\begin{array}{l}00000000000000001000000000000-11 / 2- \\
1 / 2 \\
00000000000000000100000000000-11 / 2- \\
1 / 2 \\
000000000000000000010000000000100 \\
000000000000000000000010000001-1 / 2 \\
1 / 2 \\
000000000000000000000000000010000101 \\
0000000000000000000000000001000-32-2 \\
000000000000000000000000001002-1 / 2 \\
3 / 2 \\
000000000000000000000000000000000103-22 \\
0000000000000000000000000000000001-210\end{array}$ \\
\hline 18 & $\begin{array}{l}101001001000010000010-100000-2 \mathrm{3} / 20-1 \\
3 / 2 \\
010000101100000000000100000-2 \mathrm{~g} / 4 \mathrm{3} / 2- \\
3 / 211 / 4 \\
000100000010011000000000000-2 \mathrm{~g} / 41 / 2- \\
3 / 211 / 4 \\
0000100010010100000100000000-1 / 41 / 2 \\
1 / 21 / 4 \\
00000001000000000001000000000000 \\
00000000000010000000000000005 / 41 / 2- \\
1 / 23 / 4 \\
00000000000000010000010000001 / 41 / 2- \\
1 / 23 / 4 \\
0000000000000000100000000001-3 / 4-1 / 2 \\
1 / 2-5 / 4 \\
00000000000000000100000000001 / 4-1 / 2 \\
1 / 23 / 4 \\
00000000000000000010000000003 / 21-1 \\
3 / 2 \\
00000000000000000000010000001-3 / 201- \\
3 / 2 \\
00000000000000000000000100000-1 / 41 / 2 \\
1 / 21 / 4 \\
0000000000000000000000001000010-11 \\
0000000000000000000000001000-1 / 4-1 / 2 \\
1 / 21 / 4 \\
000000000000000000000000001001 / 200 \\
1 / 2 \\
00000000000000000000000000010-1 / 41 / 2- \\
1 / 2-3 / 4\end{array}$ \\
\hline 19 & 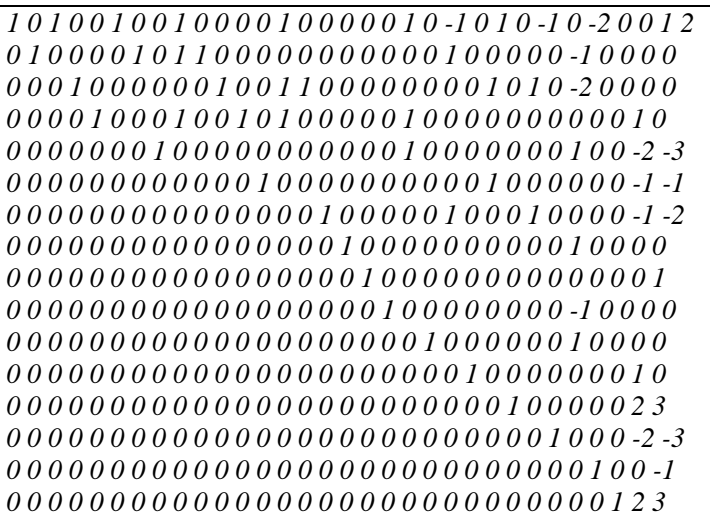 \\
\hline
\end{tabular}

Table 4: Output of $\mathrm{Md}(\mathrm{h}) *($ after Gaussian Elimination) (above).

\section{7. $\quad$ Result}

From Table 4, we can get that original Boolean function of Grain v0 do not produce any zero row in $M_{d}(h)^{*}$, that mean, it do not have any annihilator. Meanwhile only six (6) active coefficients in injected Boolean function produced zero row in $M_{d}(h) *$. All involved coefficients are as in Table 5. We bold the row zero of each $M_{d}(h) *$ in Table 4 . As for the result, all involved coefficients are as in Table 5 and we manage to obtained several annihilators using Fault Injection Attack (FAI) with HAO's algorithm.

\begin{tabular}{|c|c|}
\hline Zero row & Coefficients \\
\hline 0 row & $x_{0} x_{1}$ \\
\hline
\end{tabular}




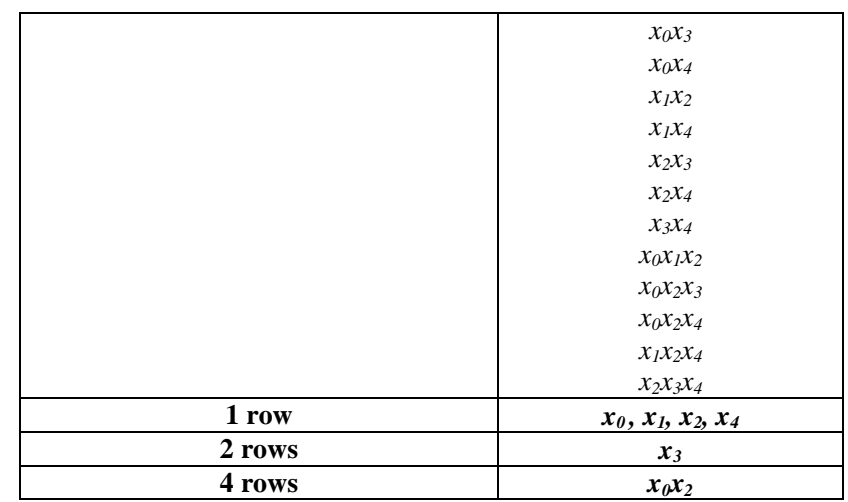

Table 5: Output of Zero row (above).

From step 3 in Algorithm 2, we manage to get annihilator via injection of coefficient $\boldsymbol{x}_{1}, \boldsymbol{x}_{\mathbf{2}}$ and $\boldsymbol{x}_{\mathbf{4}}$. Refer to Table 6 below:

\begin{tabular}{|c|c|}
\hline Coefficients & Annihilator(s) \\
\hline$x_{1}$ & $x_{2}+x_{1} x_{2}+x_{1} x_{3}+x_{2} x_{4}$ \\
\hline$x_{2}$ & $x_{0} x_{4}+x_{2} x_{4}$ \\
\hline$x_{4}$ & $x_{0} x_{1}+x_{1} x_{2}$ \\
\hline
\end{tabular}

Table 6: Output of annihilator(s) (above).

For the result comparison in this paper, by using only HAO's algorithm we do not obtained any annihilator. However, when we are using HAO's algorithm + FIA, we manage to obtain at least one (1) annihilator. Refer to Table 7 below:

\begin{tabular}{|c|c|c|}
\hline & HAO's & HAO's + FIA \\
\hline Annihilator(s) & NO & YES \\
\hline $\begin{array}{c}\text { No. of } \\
\text { Annihilator(s) }\end{array}$ & ANINIHILATOR & $\begin{array}{c}\text { THREE } \\
\text { ANINIHILATORS }\end{array}$ \\
\hline Advantage(s) & NO & $\begin{array}{c}\text { REDUCE DEGREE } \\
\text { OF ALGEBRAIC } \\
\text { ATTACK }\end{array}$ \\
\hline
\end{tabular}

Table 7: Comparison between finding annihilator(s) using only HAO’s algorithm and FIA+HAO’s algorithm (above).

\section{Conclusion}

According to $\mathrm{HAO}$, their proposed algorithm can effectively calculate all low-degree annihilators of both $h$ and $h+1$ and subsequently construct low-degree overdefined algebraic equations. But from this study, it has been found that annihilator(s) of Grain's Boolean function can be obtained only by using Fault Injection Attack (FIA) with HAO's algorithm as shown in Table 6. However, the same algorithm without FIA could not retrieve any annihilator of original Grain v0's Boolean function. This finding has concluded that HAO's algorithm is not applicable in all Boolean function based stream cipher to find an annihilator. So with our attack (HAO's algorithm + FIA), it managed to obtained annihilator(s) and help cryptanalyst to do an algebraic attack to the Boolean function based stream cipher. We also do a comparison between of this two (2) methodologies as shown in Table 7. This low-degree annihilator(s) will used to construct lowdegree overdefined algebraic equations and to be utilized in algebraic attacks. For future research, we will using the same methodology to do analysis and attack to another Boolean function based stream cipher. 


\section{References}

1. Shannon, Claude E. «Communication theory of secrecy systems. » Bell system technical journal 28.4: 656-715 (1949).

2. Hell, Martin, Thomas Johansson, and Willi Meier. «Grain : a stream cipher for constrained environments. » International Journal of Wireless and Mobile Computing 2.1: 86-93 (2007)

3. Zhang, Haina, and Xiaoyun Wang. "Cryptanalysis of Stream Cipher Grain Family." IACR Cryptology ePrint Archive 2009 (2009): 109.

4. Barenghi, A., Breveglieri, L., Koren, I., and Naccache, D. Fault injection attacks on cryptographic devices: Theory, practice, and countermeasures. Proceedings of the IEEE, 100(11):3056307.(2012).

5. Kim, Chong Hee, and Jean-Jacques Quisquater. "Faults, injection methods, and fault attacks." IEEE Design \& Test of Computers 24.6 : 544-545 (2007).

6. Guilley, Sylvain, et al. "Fault injection resilience." Fault Diagnosis and Tolerance in Cryptography (FDTC), 2010 Workshop on. IEEE, (2010).

7. Courtois, Nicolas T. "Fast algebraic attacks on stream ciphers with linear feedback." Annual International Cryptology Conference. Springer, Berlin, Heidelberg, (2003).

8. Hao, C., Shimin, W., and Zepeng, Z. Several algorithms to find annihilators of boolean function. IEEE In isdpe, pages 341-343 (2007).

9. Cusick, T. W. and Stanica, P. Cryptographic Boolean functions and applications. Academic Press.(2017).

10. Katz, Jonathan, et al. Handbook of applied cryptography. CRC press, (1996).

11. Meier, W., Pasalic, E., and Carlet, C. Algebraic attacks and decomposition of boolean functions. In International Conference on the Theory and Applications of Cryptographic Techniques, Springer pages 474-491. (2004).

12. Cao, Hao, and Huige Wang. "Constructing Boolean Functions with Maximum Algebraic Immunity." Management and Service Science (MASS), 2011 International Conference on. IEEE, 2011.

13. Cao, Hao, Hui-ge WANG, and Ze-peng ZHUO. "New Algebraic Immune Character of Boolean Function." Journal of Anhui Science and Technology University 4 : 009 (2011).

14. Banik, Subhadeep, Subhamoy Maitra, and Santanu Sarkar. "A differential fault attack on the grain family of stream ciphers." International Workshop on Cryptographic Hardware and Embedded Systems. Springer, Berlin, Heidelberg, 2012.

15. Zhang, Bin, et al. "Near collision attack on the grain v1 stream cipher." International Workshop on Fast Software Encryption. Springer, Berlin, Heidelberg, 2013.

16. Karmakar, Sandip, and Dipanwita Roy Chowdhury. "Fault analysis of Grain-128 by targeting NFSR." International Conference on Cryptology in Africa. Springer, Berlin, Heidelberg, 2011. 